\title{
Perioperative transfusion predicts poor prognosis after hepatectomy for colorectal metastases
}

\author{
Zavridis P. ${ }^{1}$, Tsitskari M. ${ }^{2}$, Petrou A. ${ }^{3}$, Neofytou K. ${ }^{4}$ \\ 1. American Medical Center, Cyprus, Dept of Anaesthesiology \& Pain Medicine, Nicosia, Cyprus \\ 2. Apollonion Private Hospital, Department of Interventional Radiology, Nicosia, Cyprus \\ 3. Nicosia General Hospital, Department of Surgery/Div. HPB, Nicosia, Cyprus \\ 4. American Medical Center, Surgery Department, Nicosia, Cyprus
}

\section{Introduction}

The combination of systemic chemotherapy and surgical resection is the treatment of choice for colorectal liver metastases (CRLM) [1]. Advances and accumulated experience in liver surgery have expanded the indications of hepatectomy in patients with CRLM, as in specialised centres the mortality of complex hepatectomies is below 5\% [2].

Hepatectomy for colorectal liver metastases can be associated with significant blood loss. Allogeneic Red Blood Cell (RBC) transfusion has been recently associated to adverse effect on survival in patients with a variety of cancers $(3,4)$. Explanations for this adverse effect are seen in the induction of the host's immunosuppression.

\section{Objectives}

The purpose of this study is to ascertain whether the perioperative transfusion affects the disease-free survival (DFS), the cancer specific survival(CSS), and overall survival (OS) of patients with liver-only colorectal metastases undergoing curative hepatectomy following neoadjuvant chemotherapy.

\section{Material \& Methods}

From our prospectively accumulated surgical database, we identified the patients who underwent liver resection for CRLM at American Medical Center and Nicosia General Hospital between January 2007 and December 2015. Inclusion criterion for this study was the preoperative administration of chemotherapy. Exclusion criteria were: a) extrahepatic disease (13 patients), b) no neoadjuvant chemotherapy (25 patients), c) incomplete resection - $\mathrm{R} 2$ resection (5 patients), d) postoperative death (1 patients), e) missing data regarding perioperative transfusion (4 patients).

For each patient the institutional electronic records was interrogated and data collected regarding: a)standard demographics, b) the primary colorectal tumour, c) the CRLM characteristics, d) the preoperative chemotherapy, e)the response to preoperative chemotherapy, f)the liver resection i)the DFS, CSS and the OS.

\section{Statistical analysis}

Statistical analysis was performed with the SPSS, v. 17.0. The end points of the study were DFS, CSS, and OS. DFS was calculated from the date of hepatectomy to the date of disease recurrence and was censored at the time of unrelated to cancer death or at the last follow up if the patients remained tumour free at those times. CSS was calculated from the date of hepatectomy to the date of cancer-related death and was censored at the time of the last follow up for patients still alive. OS was calculated from the date of hepatectomy to the date of death of any cause and was censored at the time of the last follow up for patients still alive.

Chi-square test was used for calculating the association between clinicopathologic categorical characteristics and perioperative transfusion. The impact of these features on DFS, CSS, and OS was analysed using the Kaplan-Meier method. Survival outcomes between groups were compared with the log-rank test. A P value of less than 0.05 was considered statistically significant. The factors who associated with the DFS, CSS or the OS $(\mathrm{P}>0.1)$ in univariate analysis were used for the performance of the multivariate Cox-regression analysis.

\section{Results}

A total of 122 patients were enrolled. Perioperative transfusion was used in 51 patients $(41.8 \%)$.

The median follow-up period was 36 months (2 to 102 months). During the follow-up period 83 patients $(68 \%)$ developed tumour recurrence, $44(36.1 \%)$ patients died due to progressive disease, and in total $49(40.2 \%)$ died of any cause.

The results of univariate analyses demonstrated that more than 3 liver metastases at diagnosis ( $\mathrm{HR} 1.98 ; 95 \% \mathrm{CI}, 1.21-3.24 ; \mathrm{P}=0.006$ ), bilobar distribution of lesions ( $\mathrm{HR}$ 2.22; 95\% CI, 1.40-3.57 ;P=0.001), preoperatively portal vein embolisation ( $\mathrm{HR}$ 2.87; 95\% CI, 1.46-5.65 $; \mathrm{P}=0.002)$, disease progression during neoadjuvant chemotherapy according to RECIST criteria (HR 4.06; 95\% CI, 2.10-7.88; $\mathrm{P}<0.001$ ), not administrating of adjuvant chemotherapy (HR 2.14; 95\% CI, 1.31-3.49; $\mathrm{P}=0.002$ ), and perioperative transfusion (HR 1.70; $95 \% \mathrm{CI}, 1.14-2.52 ; \mathrm{P}=0.008$ ) were associated with a decreased DFS(Table 2). Patients who were transfused showed a median DFS of 9.5 months when compared to the DFS of 17.2 months of the patients were not transfused. Three and 5-years DFS rates were 11\% and 13\% respectively in patients who were transfused and $42 \%$ and $33 \%$ respectively in patients who were not transfused.

Regarding CSS, the results of univariate analyses revealed that 5 of the analysed factors, age greater than 70 years (HR 2.18; 95\% CI, $1.15-4.12 ; \mathrm{P}=0.017$ ), more than 3 liver metastases at diagnosis (HR 1.78; $95 \%$ CI, 0.94-3.37; $\mathrm{P}=0.075$ ), bilobar distribution of lesions (HR 2.96; 95\% $\mathrm{CI}, 1.65-5.31 ; \mathrm{P}<0.001)$, not administrating of adjuvant chemotherapy (HR 3.73; 95\% CI, 2.06-6.76; $\mathrm{P}<0.001$ ), and perioperative transfusion (HR 1.90; 95\% CI, 1.67-3.12; $\mathrm{P}=0.012$ ) were associated with decreased CSS.

Multivariate analysis for DFS was adjusted for number of CRLM, distribution of CRLM, preoperatively PVE, response to neoadjuvant chemotherapy, and administration of adjuvant chemotherapy. For CSS the multivariate analysis was adjusted for age at hepatectomy, number of CRLM, distribution of CRLM, response to neoadjuvant chemotherapy, and administration of adjuvant chemotherapy. In the multivariate analysis, perioperative transfusion remained significant for DFS (HR 1.52; 95\% CI, 1.04-2.37 ;P=0.022), CSS (HR 1.67; 95\% CI, $1.09-2.85 ; \mathrm{P}=0.039$ )and OS (HR 1.76; 95\% CI, 1.09-3.68; $\mathrm{P}=0.044$ ).

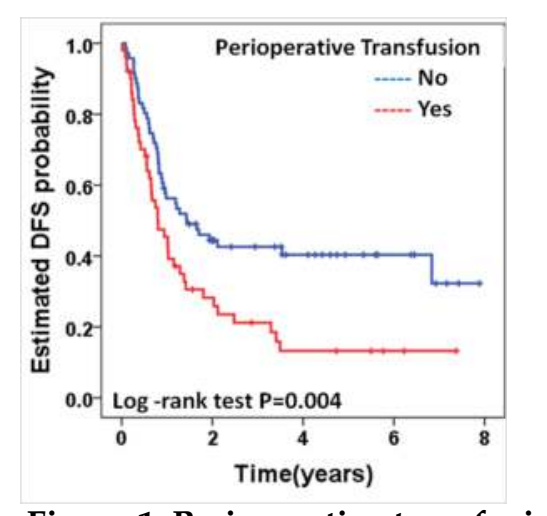

Figure 1. Perioperative transfusion and Disease Free Survival

\section{Conclusions}

In this study, we indicate that perioperative transfusion is an adverse prognostic factor in patients who undergo liver resection for colorectal metastases.

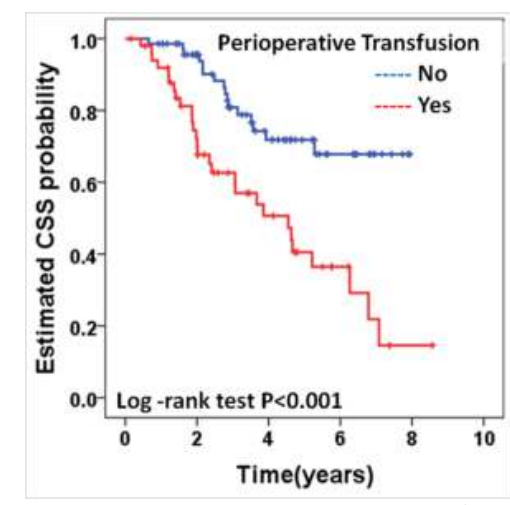

Figure 2. Perioperative transfusion and Cancer Specific Surviva

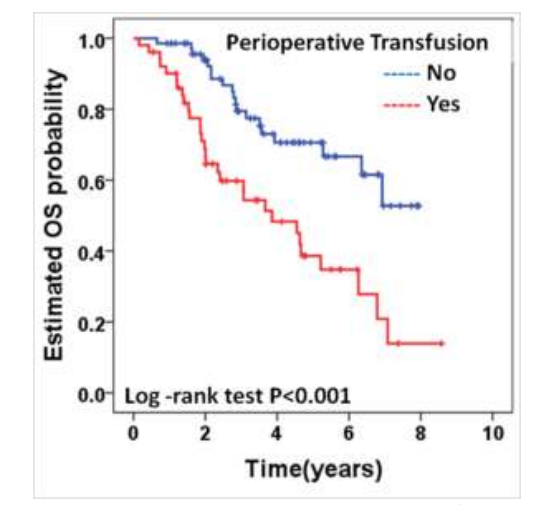

Figure 3. Perioperative transfusion and Overall Survival 